\title{
Noninvasive Localization of Premature Ventricular Activity using Different Equivalent Point Sources
}

\author{
Jana Svehlikova, Milan Tysler \\ Institute of Measurement Science, Slovak Academy of Sciences, Bratislava, Slovakia
}

\begin{abstract}
Comparison of the localization error of inverse estimation of the origin of premature ventricular contraction using three different formulations of transfer matrix between the equivalent source and the surface potentials was performed in this study.

Body surface potential maps measured in 63 precordial leads during nine spontaneous PVCs in one patient provided by Karlsruhe Institute of Technology in EDGAR database were used as input data. The localization error was evaluated with respect to two reference points $P V C 1$ and $P V C 2$ assigned during the ablation procedure. The transfer matrices for epicardial potentials, transmembrane voltages and dipoles situated on the joined endo-and-epicardial surface were used for inverse computation searching the best single point generator representing the input data

For all nine considered PVCs the locations of the inverse results were very stable - for particular transfer matrix they resulted in the same point or in adjoining points for all cases. Mean localization error with respect to PVC1 or PVC2 was from 21.3 to $26.2 \mathrm{~mm}$ and from 15.0 to $26.4 \mathrm{~mm}$ respectively.

The results obtained by inverse solution supposing single point source were similar regardless of the source formulation.
\end{abstract}

\section{Introduction}

One of the aims of electrocardiographic imaging methods (inverse methods in ECG) is the noninvasive identification of the origin of a premature ventricular contraction (PVC) [1]. Reliable results could be helpful for planning the ablation procedure used for the treatment of such heart disease. Calculation of the inverse results depends on the type of equivalent heart generator used in mathematical formulation of the problem. The reconstructed generator is usually computed in the form of epicardial potentials, transmembrane voltages or equivalent double layer [2], [3]. In [4] a single dipole was reconstructed. Because the inverse problem is ill posed, various regularization methods are tested and computational aspects are studied [5] to receive stable and physiological results. A possible improvement of the solution obtained by combining of three regularization methods was shown in [6]. The recently created EDGAR database [7] from the consortium on electrocardiographic imaging (www.ecgi-imaging.org) allows a comparison of various methods on the same data [8].

The aim of this study was to compare the single point inverse solutions assuming three types of a heart generator from the measured repeated PVCs in one patient.

\section{Materials and methods}

Measured body surface potential maps (BSPMs) were used as the input data for the inverse solution. The ECG signals were recorded in 63 precordial leads during nine spontaneous ectopic beats (PVCs) in one patient undergoing the ablation procedure [9] (patient 20). The measured dataset was provided in EDGAR database by the Institute of Biomedical Engineering, Karlsruhe Institute of Technology (KIT), Germany and the First Department of Medicine (Cardiology), University Medical Centre Mannheim, Germany. From the ablation procedure two reference points indicating possible origins of the spontaneous PVC were assigned with the CARTO system: PVC1 - the site where the earliest activation time was recorded and PVC2 - the site of the latest successful ablation.

The patient-specific geometry was obtained from MR scans taken in the end-diastolic phase and described by surface triangular meshes of the geometry of the torso and heart ventricles.

In EDGAR database the precomputed forward transfer matrices computed by boundary element method for two types of equivalent heart generators were provided: epicardial potentials (EPs) and transmembrane voltages (TMVs). The positions of the generators were assumed in the vertices of the joined triangulated endo and epicardial surface. The transfer matrix for dipoles (DIPs) situated in the same vertices was also computed.

The single point inverse solution for localization of the 
PVC origin was computed using all three equivalent heart generators (EPs, TMVs and DIPs). It was assumed that if the PVC starts in one position in the ventricle then during the initial phase of activation only small area is depolarized, that can be represented by a single equivalent heart generator. This approach was used in [4] for DIPs. In presented study the approach was used for all available generators.

To study the proper length of the initial time interval for the inverse solution, the integral BSPMs were computed for the whole PVC activation. The integral maps were computed for all time intervals from the beginning to the end of activation (1-2 ms,1-3 ms...1end).

Assume the transfer matrix $\mathrm{T}$ with size [nleads $\mathrm{x}$ nes], where nleads is the number of measuring points on the torso and nes - number of single sources in the heart. Then the BSPM can be calculated by the eq:

$$
\mathrm{BSPM}=\mathrm{T} . \mathrm{s}
$$

where $\mathrm{s}$ is a column vector of parameters of single heart sources.

Then BSPM for a single source (EP or TMV) is computed by multiplication of the $\mathrm{T}$ matrix with the corresponding value of $\mathrm{s}$ vector where parameters for all other sources are set to zero. Because dipolar source is defined by three dipolar moments the transfer matrix consists of three times more columns and the $\mathrm{s}$ vector consists of three times more parameters. BSPM from one dipole is then computed by the use of $\mathrm{T}$ matrix and the corresponding three nonzero values of the source vector moments. If only single source represents the activated heart area, for certain position of the source only one/three parameters are searched in the inverse solution. Because the input BSPM is known in 63 measured electrodes the problem leads to overdetermined system of linear equations thus can be solved by the least squares method.

The inverse solution was computed for each equivalent heart generator and each position of the single source assumed in the transfer matrix. The best position of the single heart source for each type of generator was chosen according to the criterion of minimal value of the relative difference (RELDIF) between the input BSPM and map (rec_map) reconstructed from the elementary heart source:

$$
R E L D I F=\frac{\left\|B S P M-r e c_{-} m a p\right\|}{\|B S P M\|}
$$

The localization error (LE) was computed for all types of equivalent heart generators as the Euclidean distance between the the inversely identified position of the single source generator and the both reference points PVC1 and PVC2 assigned during ablation procedure. The results for all three types of the heart generator were compared and the criteria for obtaining the best results were studied.

\section{Results}

First the parameter RELDIF was considered as a measure of the ability of the identified point heart source to represent the input integral BSPM. In Figure 1 the mean values of the RELDIF computed from the inverse results for nine repetitions (runs) of spontaneous PVCs for the first $100 \mathrm{~ms}$ of activation are shown. On the horizontal axis it is depicted the last time instant from the time interval for which the integral BSPM (as the input for the inverse solution) was computed from the beginning of the PVC. The development of the RELDIF values was similar for all three types of heart generator.

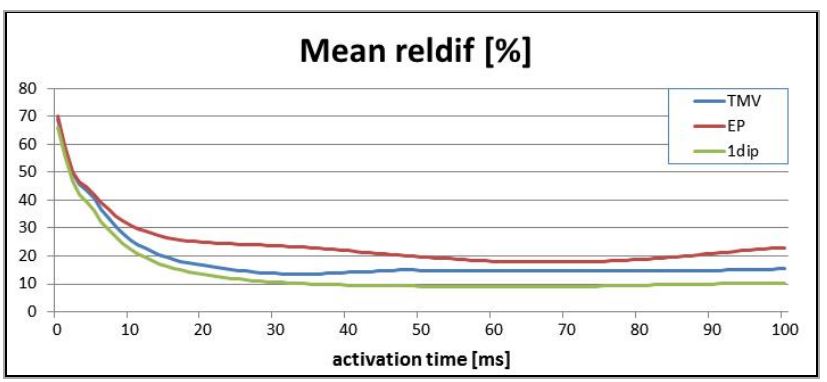

Figure 1. Mean value of RELDIF parameter for three types of point heart sources.

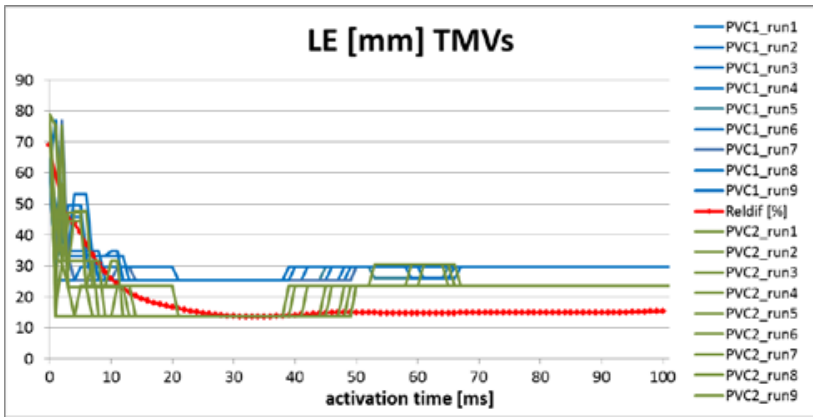

Figure 2 Localization errors with respect to the points PVC1 and PVC2 for each measured run of PVC for the heart point generator in the form of TMV. Label PVC1_run1 means that the LE with respect to PVC1 of run1 is depicted. Red line represents the corresponding mean RELDIF value in [\%].

On Figures 2, 3 and 4 the LEs computed from each run of PVC with respect to the both reference points PVC1 and PVC2 are depicted together with the corresponding course of RELDIF. When RELDIF reached its stable small value, also the locations of the inverse results were very stable. Stating that for relevant inverse results the RELDIF should not be larger than $25 \%$ and that the initial time interval for PVC can be assumed up to $25 \mathrm{~ms}$ the mean LEs with respect to PVC1/PVC2 were $25.9 \pm 1.5 \mathrm{~mm} / 15.0 \pm 3.5 \mathrm{~mm}$ for TMVs, 
$21.3 \pm 0 \mathrm{~mm} / 15.4 \pm 0 \mathrm{~mm}$ for EPs and $26.2 \pm 2.2 \mathrm{~mm} / 26.4 \pm 3.4 \mathrm{~mm}$ for DIPs. According the mentioned criteria from nine input BSPMs there were identified the relevant results for EPs in 6 cases, for TMVs and for DIPs in all 9 cases respectively.

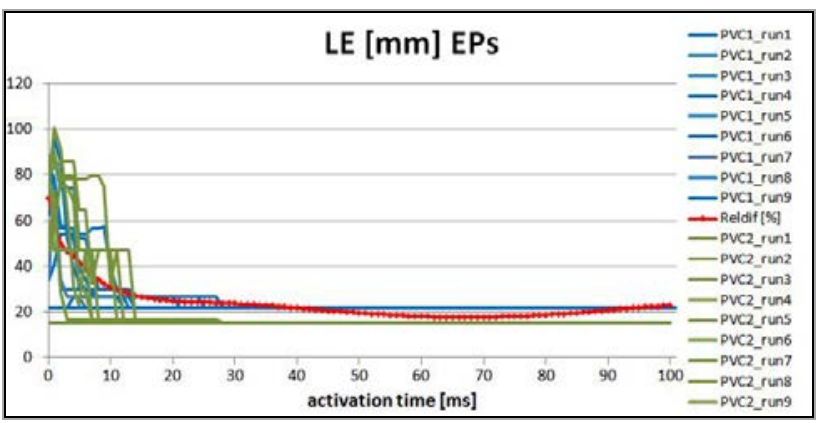

Figure 3 Localization errors with respect to the points PVC1 and PVC2 for each measured run of PVC for the heart point generator in the form of EP. Label PVC1_run1 means that the LE with respect to PVC1 of run1 is depicted. Red line represents the corresponding mean RELDIF value in [\%].

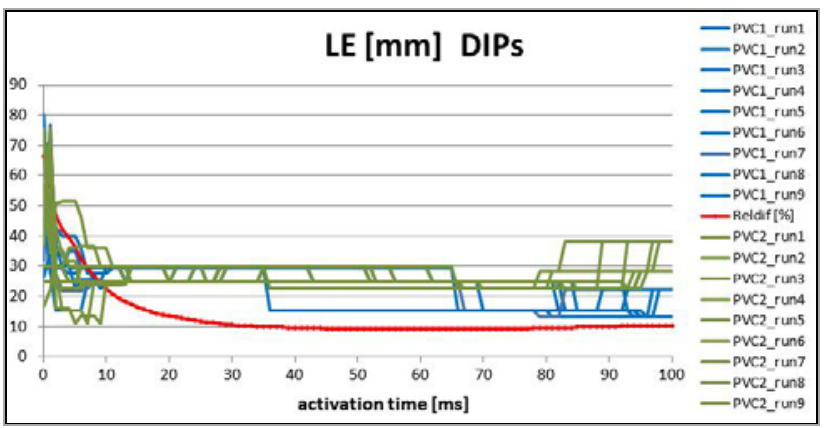

Figure 4 Localization errors with respect to the points PVC1 and PVC2 for each measured run of PVC for the heart point generator in the form of DIP. Label PVC1_run1 means that the LE with respect to PVC1 of run1 is depicted. Red line represents the corresponding mean RELDIF value in [\%].
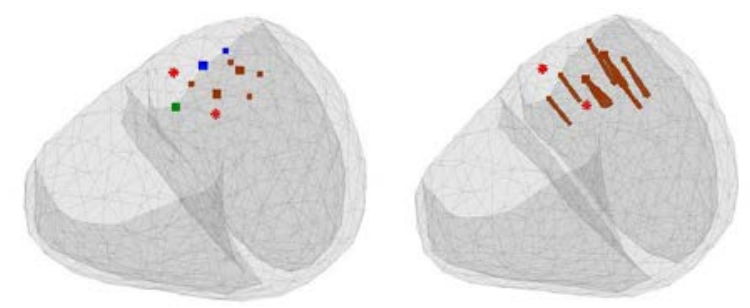

Figure 5. Left: Positions of the reference PVC origins (red stars) together with the inversely estimated PVC origins (relevant results) using TMVs (blue), EPs (green), and DIPs (brown). Right: Positions of results for DIPs with orientation of dipole moments. The points with prevalent occurrence are highlighted by larger size.
The mutual distance of the reference points PVC1 and PVC2 was $25.7 \mathrm{~mm}$. The model error i.e. the possible smallest LE achievable by the used method was $13.1 \mathrm{~mm}$ for the PVC1 and $11.2 \mathrm{~mm}$ for the PVC2.

\section{Discussion}

The presumption that BSPM measured from the PVC during the initial phase of activation can be represented by a point heart equivalent generator was applied for three types of generators. Comparison of the inverse results by RELDIF parameter showed that various single generators can represent the input BSPM with similar quality. In this case instead of regularization method a geometrical constraint was used assuming a single point source together with the rules that the value of the RELDIF parameter should not exceed 0.25 and that up to first $25 \mathrm{~ms}$ of activation the localized area is acceptable small. Of course, the parameters of such point generators did not match the physiological limits (e.g. TMV was not within $100 \mathrm{mV}$ ) but we can assume that the resulting point generator represents some small volume from which the physiological generators are summed up. It seems from the results that the point heart generator was able to represent the input integral BSPM with sufficient/acceptable quality very long time period of the PVC activation, maybe because of the only precordial positions of measuring leads or because of the specific position of the studied case of PVC.

The LE with respect to the PVC1 varied from $21.3 \pm 0 \mathrm{~mm}$ to $26.2 \pm 2.2 \mathrm{~mm}$ for all three generators and with respect to PVC2 from $15.4 \pm 0 \mathrm{~mm}$ to $26.4 \pm 3.4 \mathrm{~mm}$. Surprisingly the largest LEs were obtained for dipolar equivalent generator. There are two possible explanations. The transfer matrix for DIP was calculated using the geometry meshes from EDGAR database. Therefore the transfer matrix only for homogeneous torso model was computed. Ignoring of inhomogeneities could negatively affect the results as it was shown also in [10]. For EP and TMV generators the tranfer matrices were given by KIT group without any information what kind of torso model was used, therefore probably more apriori information was used in their computation. The second reason for the worst DIPs results could be that the physical description of the EP and TMV generator is more appropriate for such problem. On the other hand DIP solutions provide except of the position of the inversely estimated generator also the orientation of the resulting dipole as some additional information about the direction of activation propagation.

From the visual inspection of the positions of the all relevant inverse results on the heart surface it is apparent, that the position of all results was very stable. For EP generator there was only one position for all runs of PVC, for TMV generator there were only two positions (the occurrence of one was 104 out of 121 results). The largest 
variety of inverse positions seemed to be for DIP generator, however in 124 out of all relevant 137 results the dipoles were again placed in two points only. The points with dominant occurrence are highlighted by the larger size on the Figures 5 and 6.

The reference point near the aorta (PVC2) was placed outside the provided heart geometry while the first reference point PVC1 was not the place of succesfull ablation, only the place of the earliest activation time. We can doubt whether the activation time was measured also in position of PVC2. Because all obtained results were placed at the very top of the left ventricle we can hypothesize, that the reference position near the aorta was closer to the true origin of the measured spontaneous PVCs than the point PVC1.

\section{Conclusion}

The inverse solution assuming a single point equivalent heart generator was applied on BSPMs measured in 63 leads during the repeated spontaneous PVCs in one patient. The method was used for three types of generators. It was shown that in most of the PVC cases within the first $25 \mathrm{~ms}$ of activation stable results were obtained for all types of generator with relative residual error between the input BSPM and the map generated by the equivalent source less than $25 \%$. The localization error varied from 15 to $25 \mathrm{~mm}$. Now the method should be verified for different positions of the PVC origin and then could be used for the initial assessment of PVC origin position.

\section{Acknowledgements}

This work was supported by the research grant 2/0071/16 from the VEGA Grant Agency in Slovakia and by the grant APVV-14-0875 from the Slovak Research and Development Agency.

\section{References}

[1] Cluitmans MJM, Peeters RLM, Westra RL, Volders PG a. Noninvasive reconstruction of cardiac electrical activity: update on current methods, applications and challenges. Netherlands Hear J 2015;23:301-11.

[2] Potyagaylo D, Segel M, Schulze WHW, Dössel O. Noninvasive localization of ectopic foci: A new optimization approach for simultaneous reconstruction of transmembrane voltages and epicardial potentials. Lect Notes Comput Sci (Including Subser Lect Notes Artif Intell Lect Notes Bioinformatics) 2013;7945 LNCS:166-73.

[3] Van Dam PM, Tung R, Shivkumar K, Laks M. Quantitative localization of premature ventricular contractions using myocardial activation ECGI from the standard 12-lead electrocardiogram. J Electrocardiol 2013;46:574-9.

[4] Punshchykova O, Švehlíková J, Tyšler $M$, Grünes R, Sedova K, Osmančík P, et al. Influence of Torso Model Complexity on the Noninvasive Localization of Ectopic Ventricular Activity. Meas Sci Rev 2016:96-102.

[5] Tuboly G, Kozmann G, Maros I. Computational Aspects of Electrocardiological Inverse Solutions. IFACPapersOnLine 2015;48:48-51.

[6] Potyagaylo D, Schulze WHW, Olaf D. Local Regularization of Endocardial and Epicardial Surfaces for better Localization of Ectopic Beats in the Inverse Problem of ECG. Comput Cardiol 2014:10-3.

[7] Aras K, Good W, Tate J, Burton BM, Brooks DH, Coll-font $\mathrm{J}$, et al. Experimental Data and Geometric Analysis Repository - EDGAR. J Electrocardiol 2015:1-7.

[8] Coll-Font J, Erem B, Stovicek P, Brooks DH, Van Dam PM. Quantitative comparison of two cardiac electrical imaging methods to localize pacing sites. Comput Cardiol (2010) 2015;42:217-20.

[9] Schulze WHW. ECG Imaging of Ventricular Activity in Clinical Applications. PhD Thesis. Karlsruhe: KIT Scientific Publishing, Karlsruhe; 2015.

[10] Bear LR, Cheng LK, LeGrice IJ, Sands GB, Lever NA, Paterson DJ, et al. Forward problem of electrocardiography: is it solved? Circ Arrhythm Electrophysiol 2015;8:677-84.

Address for correspondence.

Jana Svehlikova

Institute of Measurement Science, Slovak Academy of Sciences Dubravska cesta 9, 84104 Bratislava, Slovakia

jana.svehlikova@savba.sk 